\title{
Pterostilbene alleviates diabetic nephropathy in experimental diabetic rats; inhibition of aldose reductase and advanced glycation end products formation
}

\begin{abstract}
Objectives: Various mechanisms including polyol pathway along with a complex integrating paradigm with oxidative stress and advanced glycation end products (AGE) formation have been implicated in the pathogenesis of diabetic nephropathy.

Methods: The present study was aimed at investigating therapeutic role of a well known antioxidant, pterostilbene in streptozotocin induced diabetic nephropathy in rats by assessing kidney function and morphological changes in kidney as the key outcome marker Fidarestat an aldose reductase (AR) inhibitor was used as reference to compare the consequences of pterostilbene on the formation of AGEs, AR inhibition and lipid peroxidation.

Results: Present study results revealed that the pterostilbene treatment diabetic rats showed decreased blood glucose levels, urinary protein excretion, serum creatinine and blood urea nitrogen significantly. And also decreased kidney lipid peroxides and nitrate levels along with reduced AGEs formation was observed. In addition, pterostilbene was found to inhibit kidney AR activity with a diminished serum transforming growth factor $\beta$ (TGF $\beta$ ) levels.

Conclusion: Thus, the results obtained in this study underline the potential of pterostilbene as a possible therapeutic agent against diabetic complications such as nephropathy.
\end{abstract}

Keywords: pterostilbene; diabetic complications; aldose reductase; diabetic nephropathy; polyol; serum; TGF $\beta$; peroxidation; angiotensin; glycation
Volume 4 Issue 4 - 2017

\author{
Dilip Dodda, Ramarao Ajmera and Ciddi \\ Veeresham \\ University College of Pharmaceutical Sciences, Kakatiya \\ University, India
}

Correspondence: Ciddi Veeresham, University College of Pharmaceutical Sciences, Kakatiya University, India, Tel +9 | 9849129584,Email ciddiveeresham@yahoo.co.in

Received: April 10, 2017 | Published: April 18, 2017
Abbreviations: AGE, advanced glycation end; AR, aldose reductase; TGF, transforming growth factor; ACE, angiotensin converting enzyme; NADPH, nicotinamide adenine dinucleotide phosphate; STZ, steptozotocin; IAEC, institutional animal ethics committee

\section{Introduction}

Diabetes mellitus has assumed epidemic proportions worldwide and such as huge burden of diabetes is sure to bring a massive burden of complications with it. Among the various diabetic complications, nephropathy (kidney disease or damage) is one of the most important, both in terms of short term and long term morbidity to the individual [1]. In spite of treating diabetic nephropathy patients with agents like angiotensin converting enzyme (ACE) inhibitors, angiotensin antagonists and antihypertensive agents, enormous number of diabetic patients still continue to suffer from diabetic kidney disease. ${ }^{2}$ Diabetic nephropathy is usually attributed to biochemical alterations in glucose metabolism such as increase in polyol flux along with elevated blood and tissue levels of glycosylated proteins leading to haemodynamic changes within the kidney tissue. ${ }^{3}$

Even though, at present, several approaches along with strict control of blood glucose level and use of ACE inhibitors for the management of diabetic kidney disease could not satisfy the clinical need for the treatment of disease which led to the research focus on alternative pathways such as aldose reductase (AR) pathway and formation of advanced glycation end products (AGEs). This led to the development of newer class of drugs which inhibit the AR an enzyme in the polyol pathway. ${ }^{4,5}$ Further, the failure of various synthetic AR inhibitors (ARIs) due to their toxic effects and absence of satisfactory efficacy, there is a pressing need for the search of newer ARIs from natural sources. ${ }^{6}$ Our preliminary studies suggested that the well known antioxidant ${ }^{7}$ phyto-constituent, pterostilbene (PST) posses a potent ARI activity with an $\mathrm{IC}_{50}$ value of $21.4 \mu \mathrm{M}$ against rat lens AR in vitro, ${ }^{8}$ which prompted us to evaluate its ability in modulating diabetic nephropathy in rats. Thus, the present study was designed to assess various biochemical and physiological alterations with special emphasis on the role of polyol pathway and AGEs in the therapy of diabetic nephropathy.

PST, it is chemically, a dimethyl ester derivative of resveratrol, is found in different plants like Vitus vinifera, Pterocarpus marsupium and $P$ santalinus. It is known to have various activities in the treatment of dyslipidemia, cardiovascular degeneration and pain. Multiple studies have demonstrated the antioxidant activity of PST in various in vitro and in vivo models. The compound has been implicated as a carcinopreventive agent and treatment of neurological disease. ${ }^{9,10}$ 


\section{Materials and methods}

\section{Chemicals}

Steptozotocin (STZ) and PST were purchased from Sigma Aldrich (Bangalore, India). Nicotinamide adenine dinucleotide phosphate (NADPH) and bovine serum albumin (BSA) were obtained from Hi Media Laboratories (Mumbai, India). Fidarestat was obtained as gift sample from Symed Labs Ltd (Hyderabad, India). All the other chemicals were of analytical grade.

\section{Animals}

Male Wistar rats (180-200 g) were procured from Sanzyme Ltd (Hyderabad, India), housed at $25^{\circ} \mathrm{C}$ and relative humidity of $45-$ $55 \%$ under a natural light: dark cycle of $12 \mathrm{~h}$ day light and $12 \mathrm{~h}$ of darkness with unrestricted access to food and water. Throughout the experimental period, the rats were fed with pellet diet has composed of $5 \%$ fat, $21 \%$ protein, $55 \%$ nitrogen-free extract and fiber $(\mathrm{w} / \mathrm{w})$ with sufficient mineral and vitamins. The experimental protocol was approved by the Institutional Animal Ethics Committee (IAEC) and executed in agreement with the guiding principles of Committee for Control and Supervision of Experimentation on Animals, Government of India on animal experimentation.

\section{Animal treatment}

Diabetes was induced by intra peritoneal administration of STZ freshly prepared in $0.1 \mathrm{M}$ citrate buffer $(\mathrm{pH} 4.5)$ to the overnight fasted rats at a dose of $50 \mathrm{mg} / \mathrm{kg}$ of body weight. Naïve animals $(\mathrm{n}=8)$ assigned as group I, received only $0.1 \mathrm{M}$ citrate buffer. The animals were then supplemented with $10 \%$ glucose solution for 48 $\mathrm{h}$ to prevent hypoglycemic shock due to administration of STZ. After one week of STZ administration, the animals were fasted for $12 \mathrm{~h}$ followed by withdrawal of blood by retro-orbital plexus. Blood glucose levels were estimated by glucose oxidase method ${ }^{11}$ and the animals with more than $250 \mathrm{mg} / \mathrm{dL}$ were treated as diabetic animals. After a period of 6 weeks, the diabetic animals were divided into 3 groups $(n=8)$ : Group II served as diabetic control group where as group III and group IV received PST $(10 \mathrm{mg} / \mathrm{kg})$ and fidarestat $(1 \mathrm{mg} /$ $\mathrm{kg}$ ) respectively, for a period of 3 weeks. The rats were allowed to get adapted to the metabolic cages for $1 \mathrm{~h} /$ day for 3 days. At the end of $3^{\text {rd }}$ day blood from retro orbital plexus and $24 \mathrm{~h}$ urine sample by means of metabolic cages were collected followed by scarifying the animals by cervical nerve dislocation. Kidneys were then perfused with normal saline, isolated, weighed and biochemical estimations were done.

\section{Biochemical estimations}

Plasma glucose was estimated enzymatically by glucose oxidase method. ${ }^{12}$ Plasma and urine creatinine were estimated by Jaffe reaction $^{13}$ and blood urea nitrogen (BUN) was estimated by the kinetic method of Wybenga et al. ${ }^{14}$ using commercially available diagnostic kits (Transasia Bio Medicals Ltd., India). Total urine protein was quantified by Bradford (1976) method using bovine serum albumin (BSA) as standard. ${ }^{15}$ Glomerular filtration rate (GFR) was calculated using $24 \mathrm{~h}$ urine volume and creatinine content in urine and plasma by the following formula: ${ }^{16}$

$\mathrm{GFR}=\frac{\text { urine creatinine concentration } \times 24 \mathrm{~h} \text { urine volume }(\mathrm{mL})}{\text { plasma creatinine concentration } \times 1440}$

\section{Estimation of AGEs in kidneys}

AGEs levels in the kidneys were determined by the method described by Sensi et al. ${ }^{17}$ Briefly, per fused kidneys were homogenized in $2 \mathrm{~mL}$ of $0.25 \mathrm{M}$ sucrose followed by centrifugation at $900 \mathrm{xg}$ at $4^{\circ} \mathrm{C}$ and the supernatant was separated. The pellet was re suspended in $2 \mathrm{~mL}$ sucrose solution and centrifuged and the supernatant obtained was mixed with the previous one. The proteins were precipitated by adding equal volume of trichloroacetic acid (TCA) then centrifuged at $4^{\circ} \mathrm{C}, 900 \mathrm{x}$ g; obtained protein pellet was mixed with $1.0 \mathrm{~mL}$ methanol twice to remove the lipid fraction. The insoluble protein fraction, after washing with $10 \%$ cooled TCA was centrifuged and the residue was solubilised in $1 \mathrm{~mL}$ of $1 \mathrm{~N} \mathrm{NaOH}$ and the protein content was estimated by colorimetric method at $280 \mathrm{~nm}$ against BSA standard curve. The AGEs content was measured flour metrically with an emission and excitation at 440 and $370 \mathrm{~nm}$ respectively, and the results were expressed as relative fluorescence units (RFU)/mg protein.

\section{Estimation of transforming growth factor (TGF- $\beta$ )}

TGF- $\beta$ levels in the serum were determined by using ELISA kit (R\&D systems, Inc. USA). The analysis was performed according to the manufacturer's instructions. Standard plots were constructed using standard TGF- $\beta$ and the concentrations of unknown samples were calculated from the standard plot.

\section{Histological study}

A portion of the kidney tissue was fixed in $10 \%$ formalin for 7 days at room temperature. Then the specimens were dehydrated with graded ethanol, cleared in xylene and embedded in paraffin wax. The blocks were sectioned into $5 \mu \mathrm{m}$ thick using rotary microtome. The obtained sections were stained with hematoxylin-eosin and the photomicrographs were obtained under light microscope at a magnification of 400x and analyzed by double blind analysis and scored by the method of Qi and Wu. ${ }^{18}$ Tubular injury was scored by grading the percentage of affected tubules under ten randomly selected, non-overlapping fields as follows: $0,0 \% ; 1, \leq 10 \% ; 2,11-25 \% ; 3,26-$ $45 \%$; 4, 46-75\%; and 5, 76-100\%. To score injured tubules, whole tubule numbers per field were considered as standard. The grading percentage was calculated in each field as follows: Injury score (\%) = (number of injured tubules/number of whole tubules) x100

\section{Kidney AR activity}

AR activity was measured spectrophotometrically by the earlier reported method of Kim and $\mathrm{Oh}^{19}$ Briefly, the reaction mixture consisted of $300 \mu \mathrm{L}$ of $0.15 \mathrm{mM}$ NADPH, crude enzyme preparation, and the final volume was made up to $2.7 \mathrm{~mL}$ with sodium phosphate buffer. The reaction was initiated by addition of $300 \mu \mathrm{L}$ of $10 \mathrm{mM}$ DL-glyceraldehyde as substrate and absorbance was measured at 340 $\mathrm{nm}$ using double beam UV spectrophotometer (SL210, Elico, India) for $1 \mathrm{~min}$ at $10 \mathrm{sec}$ interval. Absorbance was recorded for all the concentrations in triplicate.

\section{TBARS content}

TBARS content was estimated by the method of Utley et al..$^{20}$ Briefly, $0.25 \mathrm{~mL}$ of kidney homogenate was incubated at $37^{\circ} \mathrm{C}$ in an incubator shaker for $1 \mathrm{~h}$. And same volume of the homogenate was placed at $0^{\circ} \mathrm{C}$ for $1 \mathrm{~h}$. After $1 \mathrm{~h}$ incubation, $0.5 \mathrm{~mL}$ of $5 \%(\mathrm{w} / \mathrm{v})$ cold trichloroacetic acid (TCA) was added followed by addition of 
$0.5 \mathrm{~mL}$ of $0.67 \% \mathrm{TBA}(\mathrm{w} / \mathrm{v})$ and centrifuged at $900 \mathrm{x} \mathrm{g}$ for $20 \mathrm{~min}$. The supernatant obtained was then heated in water bath for $10 \mathrm{~min}$. The TBARS quantity was then calculated by using the extinction coefficient of $1.56 \times 10^{5} / \mathrm{M} / \mathrm{cm}$ at $535 \mathrm{~nm}$. The TBARS content was expressed as nmol formed per minute per $\mathrm{mg}$ of protein.

\section{Serum and urinary nitrate levels}

Serum and urine nitrate levels were estimated according to the method described by Miranda et al., ${ }^{21}$ Nitrite and sulphonamide react with $\mathrm{N}$-(1-napthyl) ethylenediamine to form an azo product which was quantified by measuring the absorbance of the product at $543 \mathrm{~nm}$. The concentrations were determined using a standard curve of sodium nitrate and the results were expressed as $\mu \mathrm{mol} / \mathrm{l}$ in serum and nmol/ min in urine samples.

\section{Statistical analysis}

The data was analyzed by using analysis of variance (ANOVA) followed by Bonferroni post test. All the values were expressed as mean \pm SEM and the criterion for statistical significance was considered to be $\mathrm{P}<0.05$.

\section{Results}

\section{Effect on blood glucose}

Intra peritoneal administration of STZ led to a significant $(\mathrm{P}<0.05)$ increase in blood glucose levels in the control group when compared to the naïve animals. However, administration of PST $(10 \mathrm{mg} / \mathrm{kg})$ for 21 days led to a significant $(\mathrm{P}<0.05)$ decrease in the blood glucose when compared to the control group, whereas, administration of fidarestat $(1 \mathrm{mg} / \mathrm{kg})$ for 3 weeks did not show any significant decrease in blood glucose when compared to control group animals (Table 1).

\section{Renal function related parameters}

Urinary protein, plasma creatinine and BUN were significantly increased in the diabetic control group when compared to naïve animals. However, administration of PST $(10 \mathrm{mg} / \mathrm{kg})$ and fidarestat $(1 \mathrm{mg} / \mathrm{kg})$ for 21 days led to a significant decrease in the urinary albumin, plasma creatinine and BUN when compared to the control group (Table 1). Further, creatinine levels of urine in control group decreased significantly $(\mathrm{P}<0.05)$ when compared to naïve animals, which was significantly increased by the administration of PST or fidarestat. Similarly, GFR was also found to be significantly $(\mathrm{P}<0.05)$ decreased in control group when compared to the naïve group animals. Administration of PST or fidarestat for 3 weeks led to a significant $(\mathrm{P}<0.05)$ increase in the GFR when compared to the control group.

\section{Effect of PST on kidney AGEs and AR}

Inductions of diabetic nephropathy in rats led to a significant enhance AGEs levels in kidney when compared to naïve rats. Administration of PST $(10 \mathrm{mg} / \mathrm{kg})$ reduced the AGEs levels in kidneys significantly $(\mathrm{P}<0.05)$ when compared to control group. However, administration of fidarestat $(1 \mathrm{mg} / \mathrm{kg})$ produced an insignificant decrease in AGEs levels when compared to control group (Figure 1a). Similarly, the activity of kidney AR was significantly increased in diabetic rats compared to the normal rats. Administrations of PST or fidarestat oppose the AR activity to a significant $(\mathrm{P}<0.05)$ extent when compared to the diabetic rats (Figure 1b).

Table I Biochemical parameters in STZ induced diabetic nephropathy in rats

\begin{tabular}{|c|c|c|c|c|}
\hline Parameters & Naive & Control & Diabetic + PST & Diabetic + Fidarestat \\
\hline Blood glucose (mg/dl) & $104 \pm 4.37$ & $572 \pm 16.83^{\#}$ & $277 \pm 8.64 *$ & $524 \pm 28.35$ \\
\hline BUN (mg/dl) & $15.35 \pm 1.04$ & $69.35 \pm 0.97^{\#}$ & $24.29 \pm 2.17 *$ & $18.22 \pm 1.30 *$ \\
\hline Urine protein (mg/24h) & $24.48 \pm 1.95$ & $82.19 \pm 3.17^{\#}$ & $36.24 \pm 1.70^{*}$ & $32.80 \pm 1.48 *$ \\
\hline Serum creatinine $(\mathrm{mg} / \mathrm{dl})$ & $0.57 \pm 0.07$ & $\mathrm{I} .45 \pm 0.1 \mathrm{I}^{\#}$ & $0.87 \pm 0.06^{*}$ & $0.72 \pm 0.12 *$ \\
\hline Urine Creatinine (mg/dl) & $1.13 \pm 0.13$ & $0.33 \pm 0.04^{\#}$ & $0.85 \pm 0.05 *$ & $0.97 \pm 0.06 *$ \\
\hline GFR (ml/min) & $2.6 I \pm 0.5 I$ & $0.91 \pm 0.07^{\#}$ & $1.58 \pm 0.18^{*}$ & $1.83 \pm 0.32 *$ \\
\hline
\end{tabular}

Data was analyzed by one Way ANOVA followed by Dunnet's test. Values are expressed as mean \pm SEM. $(n=8) \# P<0.05$ as compared to naive group, $* P<0.05$ as compared to control group.

\section{Effect of PST on TGF- $\beta$}

Serum TGF- $\beta$ levels was significantly increased in diabetic control group when compared to naive animals (Figure 1c). When the diabetic rats were treated with PST $(10 \mathrm{mg} / \mathrm{kg})$ or fidarestat $(1 \mathrm{mg} / \mathrm{kg})$ there was a significant decrease in serum level of TGF- $\beta$ compared to the control group.

\section{Histology}

Tissue sections study by Light microscopic revealed that normal rats consisted of intact glomeruli with normal mesangial matrix.
However, diabetic rats exhibited glomeruli with mesangial spreading out which is reflected by the changes in histopathological scoring and loss of some podocyte cells in diabetic rats. However administration of PST $(10 \mathrm{mg} / \mathrm{kg})$ or fidarestat $(1 \mathrm{mg} / \mathrm{kg})$ led to the reduction in mesangial spreading out and prevention of loss of podocyte cells (Figure 2).

\section{Effect of PST on TBARS and serum/urine nitrate levels}

TBARS levels were significantly $(P<0.05)$ high in whole kidney tissues of diabetic untreated rats when compared to naïve animals. 
Treatment with PST $(10 \mathrm{mg} / \mathrm{kg})$ or fidarestat $(1 \mathrm{mg} / \mathrm{kg})$ significantly reverted the TBARS content in renal tissues. Similarly, total nitrite and nitrate levels in both serum and urine were significantly $(P<0.05)$ elevated in the diabetic untreated group when compared to naïve animal's results. However, treatment with PST or fidarestat at a dose of $10 \mathrm{mg} / \mathrm{kg}$ and $1 \mathrm{mg} / \mathrm{kg}$ respectively, inhibited the diabetes induced raise in nitrate levels as compared to the untreated diabetic rats (Figure 3).
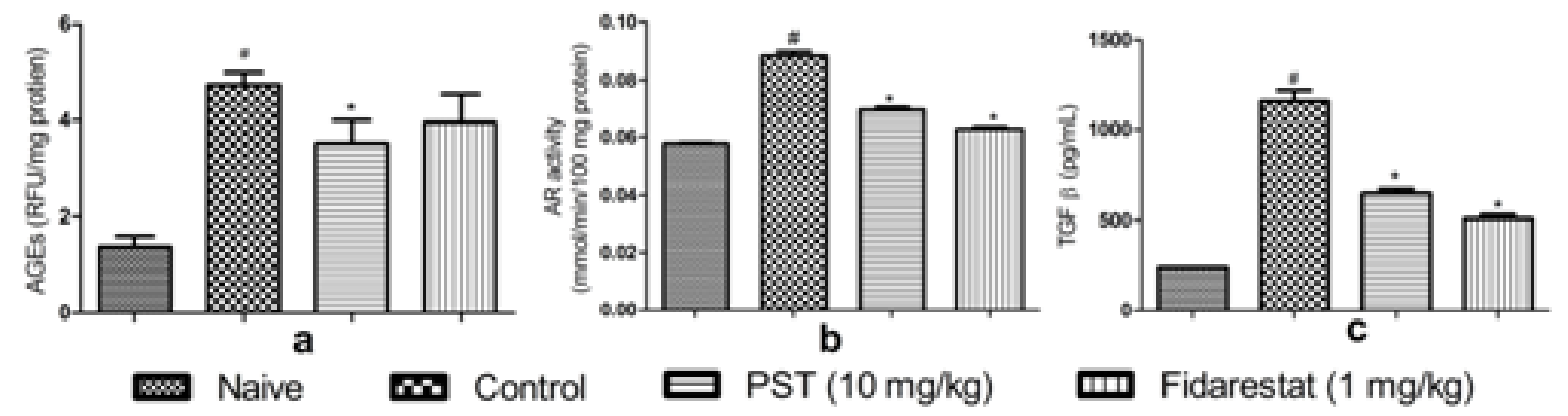

Figure I Effect of PST on alterations in (a) AGE levels (b) AR activity and (c) serum TGF $\beta$ levels in STZ induced diabetic nephropathy in rats. RFU: Relative

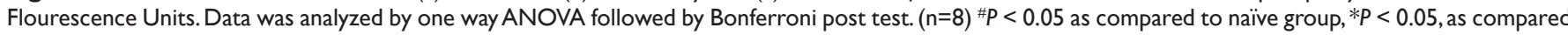
to control group.
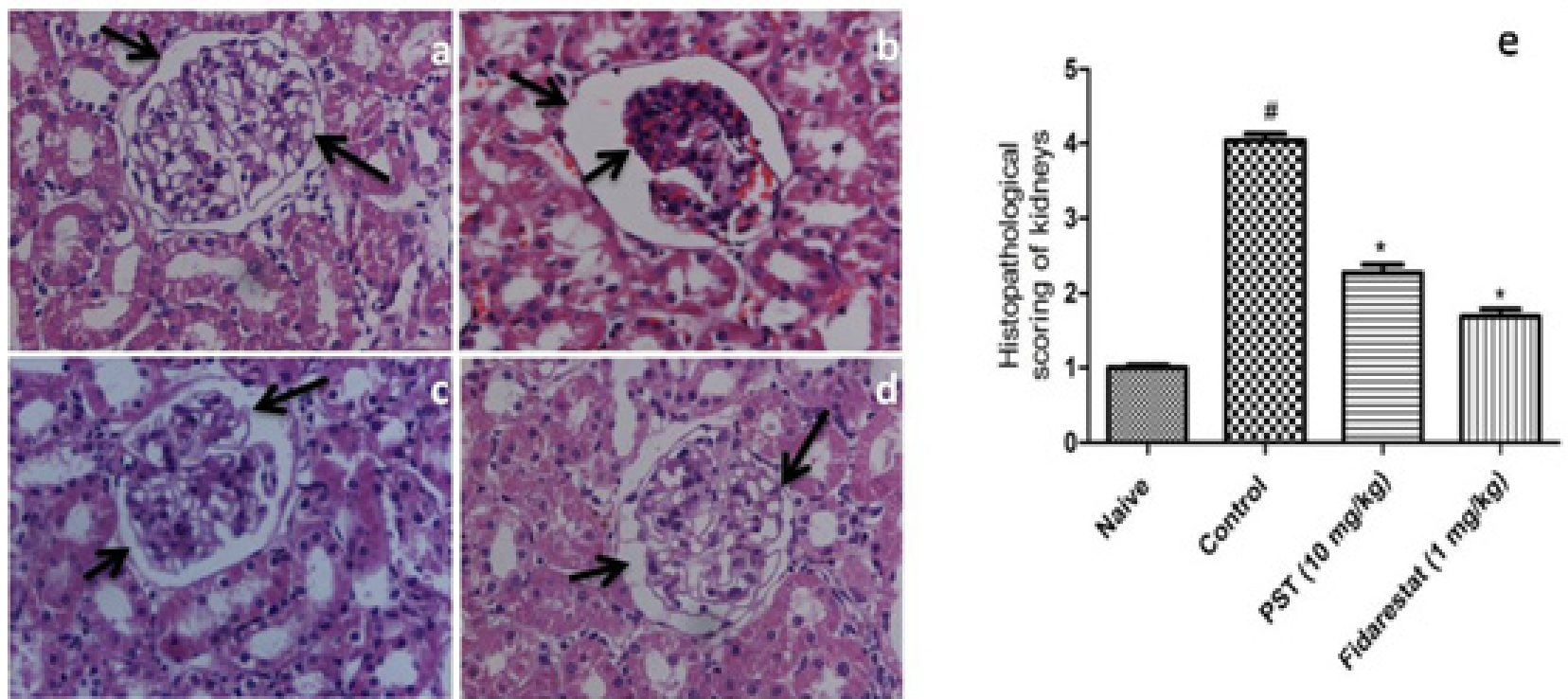

Figure 2 Glomerular morphology changes in different experimental groups (HE stain, 400x magnification). (a) Naive group (b) Control group (c) Diabetic + PST (d) Diabetic + Fidarestat (e) Histopathological scoring of STZ-induced renal injury. Data was analyzed by one way ANOVA followed by Bonferroni post test. $(n=8){ }^{\# P}<0.05$ as compared to naïve group, $* P<0.05$, as compared to control group.

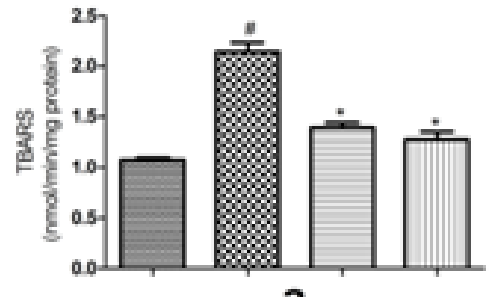

a

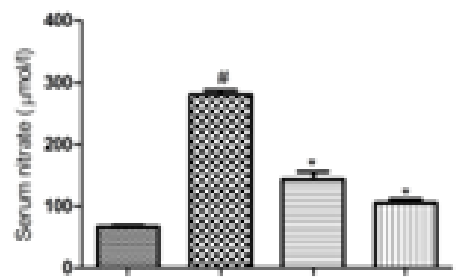

b

PST $(10 \mathrm{mg} / \mathrm{kg}$ )

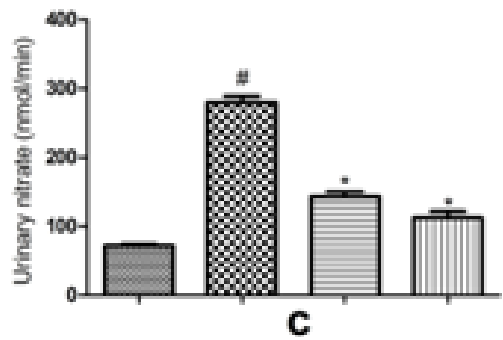

m Fidarestat (1 mg/kg)

Figure 3 Effect of PST on (a) TBARS content (b) Serum nitrate levels and (c) Urinary nitrate levels in STZ induced diabetic nephropathy in rats. Data was analyzed by one way ANOVA followed by Bonferroni post test. $(n=8){ }^{\#} P<0.05$ as compared to naïve group, $* P<0.05$, as compared to control group. 


\section{Discussion}

A large number of studies have documented the evidence that progression of diabetes leads to various secondary diabetic complications among which nephropathy is a serious complication with an increasing prevalence worldwide. ${ }^{22}$ The nephropathy disease is characterized by morphological and ultra structural changes in the kidney including expansion of the molecular matrix. Even though, the pathogenesis of diabetic nephropathy is complex and still not fully elucidated, few biochemical changes such as increase in polyol pathway flux, increased AGEs formation, have been actively studied for their role in the development of diabetic nephropathy. Increased matrix proteins leading to decreased GFR is considered as a marker for the progression of the Diabetic nephropathy disease. Elevation of serum creatinine levels and BUN in diabetic rats is used as an index of altered GFR in diabetic nephropathy. ${ }^{23}$

Results of the present study have corroborated with the previous reports in which administration of STZ led to the induction of diabetic nephropathy with a significant decrease in GFR which is evident from the elevated levels of serum creatinine and BUN. ${ }^{24}$ However, administration of PST for 21 days resulted in improved kidney function by normaling kidney parameters like creatinine and BUN along with increased GFR in diabetic rats. Even though the fidarestat treatment did not restore the glucose levels in diabetic rats, it led to a significant decrease in the UFR to normal levels (evident from the increased GFR) which can be attributed to its potent ARI and antioxidant activity. As the consequence of increased GFR, excretion of proteins in urea (protienurea) as a result of decreased tubular re absorption becomes the vital marker in diabetic nephropathy. ${ }^{25}$ In consistent with the above findings, the present study exhibited higher urine protein levels in diabetic rats when compared to naïve group. On the other hand PST treated group significantly reduced the degree of proteinuria.

Activity of $\mathrm{AR}$ is accelerated in hyperglycemia condition to convert the glucose to sorbitol with a parallel decrease in NADPH and glutathione. Thus the inequity of these antioxidants results in increased susceptibility to oxidative stress allied with intracellular reactive oxygen species. ${ }^{26}$ As an effect of accumulation of impermeable sorbitol, increased glycation of intracellular proteins takes place, lead to the increased AGEs formation. Increased AR activity triggers various signal transduction pathways such as PKC and JNK resulting in the overproduction of cytokines such as TGF- $\beta$. Several earlier reported studies were evidence for the elevation of AR in the renal tissue during diabetes and a favorable influence of ARIs in diabetic nephropathy. ${ }^{27}$

Inferences from the preliminary studies on PST as a potent ARI in vitro (unpublished results) was well thought-out to evaluate for its therapeutic efficacy in diabetic nephropathy. In the present study, AR activity was significantly increased in diabetic rats, which was inhibited by administration of PST. Consequently, the in vivo ARI potential of PST was established in the study. Further, the levels of AGEs formation in the kidneys were estimated, for the reason that mentioned earlier i.e., AGEs play a key role in the progress of diabetic nephropathy. ${ }^{28}$ Decreased levels of AGEs after PST administration to diabetic rats indicative its beneficial role in therapy of the disease. These results supports the earlier report by Lv et al., ${ }^{29}$ where PST effectively interrupt the formation of AGEs by trapping of methylglyoxal, a reactive diacarbonyl intermediate. Further, administration of fidarestat led to an insignificant decrease in the
AGEs which can be attributed to the high glucose levels, never the less, the potent AR inhibitory activity of the drug along with its ability to inhibit the oxidative stress in the rats could have contributed to the normalization of the kidney function in the diabetic rats.

Several studies involving both preclinical and clinical studies suggest that oxidative stress play a vital role in the development of diabetic complications. In hyperglycemic condition induces free radical generation, combined with AR mediated decrease in NADPH and glutathione leads to oxidative stress. ${ }^{30}$ TBARS, which is a marker of lipid peroxidation in kidneys, was increased in the diabetic untreated rats and this increase in TBARS was significantly decreased by administration of PST. Moreover, nitrate was also considered as an index of oxidative damage since these are oxidation products of $\mathrm{NO} .{ }^{31}$ In the present investigation, we observed that the serum and urine nitrate levels in PST or fidarestat treated animals were significantly decreased demonstrating its protective role against STZ-induced diabetic complications. This effect can be attributed to its antioxidant activity along with its ability to inhibit polyol pathway induced oxidative stress. Early study reported that the therapeutic role of PST in STZ-nicotinamide induced diabetic rat which was attributed to its antioxidant and antihyperglycemic activity. ${ }^{9}$

In line with the above findings, administration of PST to the diabetic rats led to a significant decrease in blood glucose along with the decreased oxidative stress in rats. TGF- $\beta$ plays an important role in extracellular matrix (ECM) metabolism. Several studies have reported that various stimuli such as hyperglycemia, AGEs and oxidative stress increase TGF- $\beta$ production, lead to excessive amount of ECM, followed by glomerular fibrosis results ultimately in loss of renal function. ${ }^{11,32}$ Biochemical analysis by ELISA and histopathological studies of the kidney sections showed a significant increase in TGF- $\beta$ levels in diabetic rats with concomitant mesangial expansion and deterioration of renal function. The accumulation of TGF- $\beta$ and histological changes in diabetic rats was also inhibited by PST.

In conclusion, the therapeutic potential of PST against STZ induced diabetic nephropathy can be attributed to its combined effect of its ability to inhibit AR and AGEs formation along with its well known antioxidant and antihyperglycemic effects. However further studies in diabetic model with knockout AR rats are necessary to confirm the exact mechanism of action along with its effect on various other diabetic complications.

\section{Acknowledgements}

The authors are thankful to University Grants Commission, Government of India for the financial support in the form of UGCBSR fellowship to one of the authors. A.R.R gratefully acknowledges the award of Post Doctoral fellowship by the UGC, Government of India. Symed Labs Ltd (Hyderabad, India) is gratefully acknowledged for the generous gift of fidarestat sample.

\section{Conflicts of interest}

The author declares there is no conflict of interest.

\section{References}

1. Rossing P. Diabetic nephropathy: worldwide epidemic and effects of current treatment on natural history. Curr Diab Rep. 2006;6(6):479683. 
2. Dronavalli S, Duka I, Bakris GL. The pathogenesis of diabetic nephropathy. Nat Clin Pract Endocrinol Metab. 2008;4(8):444-452.

3. Aurell M, Björck S. Determinants of progressive renal disease in diabetes mellitus. Kidney Int Suppl. 1992;36:S38-S42.

4. Jennings PE, Nightingale $\mathrm{S}$, Le Guen $\mathrm{C}$, et al. Prolonged aldose reductase inhibition in chronic peripheral diabetic neuropathy: effects on microangiopathy. Diabet Med. 1990;7(1):63-68.

5. Lewis EJ, Hunsicker LG, Bain RP, et al. The effect of angiotensinconverting-enzyme inhibition on diabetic nephropathy. The Collaborative Study Group. N Engl J Med. 1993;329(20):1456-1462.

6. Veeresham C, Rama Rao A, Asres K. Aldose reductase inhibitors of plant origin. Phytother Res. 2014;28(3):317-333.

7. Chakraborty A, Gupta N, Ghosh $\mathrm{K}$, et al. In vitro evaluation of the cytotoxic, anti-proliferative and anti-oxidant properties of pterostilbene isolated from Pterocarpus marsupium. Toxicol In Vitro. 2010;24(4):1215-1228

8. Dilip D, Veeresham C (2015) Pterostilbene alleviates diabetic nephropathy in experimental diabetic rats; inhibition of aldose reductase and advanced glycation end products formation. Oriental Pharmacy and Experimental Medicine 15(4): 297-303.

9. Amarnath Satheesh M, Pari L (2006) The antioxidant role of pterostilbene in streptozotocin-nicotinamide-induced type 2 diabetes mellitus in Wistar rats. J Pharm Pharmacol 58(11): 1483-1490.

10. McCormack D, McFadden D (2013) A review of pterostilbene antioxidant activity and disease modification. Oxid Med Cell Longev 2013: 575482

11. Forbes JM, Fukami K, Cooper ME (2007) Diabetic nephropathy: where emodynamics meets metabolism. Exp Clin Endocrinol Diabetes 115(2): 69-84.

12. Huggett AS, Nixon DA (1957) Use of glucose oxidase, peroxidase, and $\mathrm{O}$-dianisidine in determination of blood and urinary glucose. Lancet 273(6991): 368-370

13. Hervey GR (1953) Determination of creatinine by Jaffe reaction. Nature 171(4364): 1125

14. Wybenga DR, Di, Giorgio J, Pileggi VJ (1971) Manual and automated methods for urea nitrogen measurement in whole serum. Clin Chem 17(9): 891-895.

15. Bradford MM (1976) A rapid and sensitive method for the quantitation of microgram quantities of protein utilizing the principle of protein-dye binding. Anal Biochem 72: 248-254.

16. Baig MA, Gawali VB, Patil RR, Naik SR (2012) Protective effect of herbomineral formulation (Dolabi) on early diabetic nephropathy in streptozotocin-induced diabetic rats. J Nat Med 66(3): 500-509.

17. Sensi M, Pricci F, Pugliese G, De Rossi MG, Petrucci AF, et al. (1996) Role of advanced glycation end-products (AGE) in late diabetic complications. Diabetes Res Clin Pract 28(1): 9-17.
18. Qi S, Wu D (2013) Bone marrow-derived mesenchymal stem cells protect against cisplatin-induced acute kidney injury in rats by inhibiting cell apoptosis. Int J Mol Med 32(6): 1262-1272.

19. Kim HY, Oh JH (1999) Screening of Korean forest plants for rat lens aldose reductase inhibition. Biosci Biotech Biochem 63(1): 184-188.

20. Utley HG, Frederick B, Paul H. Effect of sulfhydryl reagents on peroxidation in microsomes. Archives of Biochemistry and Biophysics. 1967;118:29-32.

21. Miranda K, Espy MG, Wink DA. A rapid and simple spectrophotometric method for simultaneous detection of nitrate and nitrite. Nitric Oxide. 2001;5(1):62-71

22. Packham DK, Alves TP, Dwyer JP, et al. Relative incidence of ESRD versus cardiovascular mortality in proteinuric type 2 diabetes and nephropathy: results from the DIAMETRIC (Diabetes Mellitus Treatment for Renal Insufficiency Consortium) database. Am J Kidney Dis. 2012;59(1):75-83.

23. Rudberg S, Persson B, Dahlquist G. Increased glomerular filtration rate as a predictor of diabetic nephropathy - an 8-year prospective study. Kidney Int. 1992;41(4):822-828.

24. Jiang WL, Zhang SP, Hou J, et al. Effect of loganin on experimental diabetic nephropathy. Phytomedicine. 2012;19(3-4):217-222.

25. Maack T, Johnson V, Kau ST, et al. Renal filtration, transport, and metabolism of low-molecular-weight proteins: a review. Kidney Int. 1979;16(3):251-270.

26. Brownlee M. Biochemistry and molecular cell biology of diabetic complications. Nature. 2001;414(6865):813-820.

27. Oates PJ, Mylari BL. Aldose reductase inhibitors: therapeutic implications for diabetic complications. Expert Opin Investig Drugs. 1999;8(12):2095-2119.

28. Forbes JM, Cooper ME, Oldfield MD, et al. Role of advanced glycation end products in diabetic nephropathy. J Am Soc Nephrol. 2003;14(8 Suppl 3):S254-S258.

29. Lv L, Shao X, Wang L, et al. Stilbene glucoside from Polygonum multiflorum Thunb: a novel natural inhibitor of advanced glycation end product formation by trapping of methylglyoxal. J Agric Food Chem. 2010;58(4):2239-2245.

30. Baynes JW, Thorpe SR. Role of oxidative stress in diabetic complications: a new perspective on an old paradigm. Diabetes. 1999;48(1):1-9

31. Pitocco D, Zaccardi F, Romitelli F, et al. Oxidative stress, nitric oxide, and diabetes. Rev Diabet Stud. 2010;7(1):15-25.

32. Nishibayashi S, Hattori K, Hirano T, et al. Functional and structural changes in end-stage kidney disease due to glomerulonephritis induced by the recombinant alpha3 (IV)NC1 domain. Exp Anim. 2010;59(2):157-170. 